\title{
ESTABELECIMENTO DE NORMAS DRIS EM CAFEEIRO CONILON ORGÂNICO OU CONVENCIONAL NO ESTADO DO ESPÍRITO SANTO ${ }^{(1)}$
}

\author{
Fabio Luiz Partelli ${ }^{(2)}$, Henrique Duarte Vieira ${ }^{(3)}$, Pedro Henrique \\ Monnerat $^{(3)}$ \& Alexandre Pio Viana ${ }^{(3)}$
}

\begin{abstract}
RESUMO
O café conilon (Coffea canephora) ocupa grande importância no cenário sócioeconômico no Espírito Santo, e o seu cultivo, principalmente, de forma orgânica é crescente e representativo; no entanto, faltam informações sobre valores de referência de teores da folha, bem como um diagnóstico nutricional. $O$ objetivo deste trabalho foi estabelecer e comparar padrões de referência e propor valores de teores foliares considerados adequados, a partir de lavouras de alta produtividade de cafeeiros conilon cultivados de forma orgânica ou convencional na região norte do estado do Espírito Santo. Determinaram-se os teores foliares de $\mathrm{N}, \mathrm{P}, \mathrm{K}, \mathrm{Ca}, \mathrm{Mg}, \mathrm{S}, \mathrm{B}, \mathrm{Cu}, \mathrm{Fe}$, Mn e Zn em 40 lavouras sob cultivo convencional e 56 lavouras sob cultivo orgânico. Foram selecionadas para estabelecer as normas DRIS 23 lavouras sob cultivo convencional e 22 lavouras orgânicas. Para comparar as normas estabelecidas, utilizou-se o teste "t" de Student, a $1 \%$, e realizaram-se diagnósticos nas lavouras orgânicas e convencionais, por meio das normas DRIS, com a incorporação da matéria seca, verificando a freqüência do nutriente cujo índice foi mais negativo e mais positivo. Os nutrientes que mais vezes apresentaram índices DRIS mais negativos nas lavouras orgânicas foram $\mathrm{Mn}, \mathrm{Cu}, \mathrm{N}$ e $\mathrm{P}$ e, nas lavouras convencionais, foram $\mathrm{Mn}, \mathrm{Cu}$ e $\mathrm{P}$. Os resultados evidenciam que as normas DRIS são específicas a cada forma de cultivo.
\end{abstract}

Termos de indexação: Coffea canephora, DRIS, diagnóstico nutricional, equilíbrio nutricional.

\footnotetext{
(1) Parte da Tese de Mestrado do primeiro autor. Recebido para publicação em agosto de 2004 e aprovado em março de 2006.

(2) Engenheiro-Agrônomo, Doutorando em Produção Vegetal, Universidade Estadual do Norte Fluminense Darcy Ribeiro UENF. Av. Alberto Lamego 2000, CEP 28013-602 Campos dos Goytacazes (RJ). E-mail:partelli@yahoo.com.br

(3) Professor do Centro de Ciências e Tecnologias Agropcuárias, UENF. E-mails:henrique@uenf.br; monnerat@uenf.br; pirapora@uenf.br
} 


\title{
SUMMARY: ESTABLISHMENT OF DRIS NORMS FOR ORGANIC AND CONVENTIONAL CONILON COFFEE IN THE STATE OF ESPIRITO SANTO (BRAZIL)
}

\begin{abstract}
Conilon coffee (Coffea canephora) is very important for the economy of the state of Espirito Santo and its organic cultivation is increasing. However, there is a lack of information on leaf reference values for this form of management, as well as a nutritional diagnosis. The objective of this study was to establish and compare reference patterns and to propose adequate values of leaf nutrient concentrations obtained on high yielding conilon coffee plantations, cultivated under organic or conventional systems, in the northern area of Espirito Santo State. Leaf concentrations of $\mathrm{N}, \mathrm{P}, \mathrm{K}, \mathrm{Ca}, \mathrm{Mg}, \mathrm{S}, \mathrm{B}, \mathrm{Cu}, \mathrm{Fe}, \mathrm{Mn}$, and $\mathrm{Zn}$ were determined in plantations under conventional and 56 plantations under organic cultivation. To establish DRIS norms, the data of 23 plantations under conventional cultivation and 22 organic plantations were used. The established norms were compared by the Student " $t$ " test at $1 \%$ probability. Organic and conventional plantations were diagnosed through DRIS norms with the incorporation of dry matter data in the analysis. The frequency of the nutrients with the most negative or most positive index was verified. The most frequent nutrients with more negative DRIS index on the organic plantations were $\mathrm{Mn}, \mathrm{Cu}, \mathrm{N}$, and $\mathrm{P}$. In the conventional plantations, the most frequent nutrients with more negative index were $M n$, Cu and $P$. The results suggested that each cultivation system should be diagnosed by specific DRIS norms.
\end{abstract}

Index terms: Coffea canephora, DRIS, nutritional diagnosis, nutritional balance.

\section{INTRODUÇÃO}

O diagnóstico nutricional de uma planta e, ou, lavoura depende de valores de referência, tais como: nível crítico e faixa ótima para a concentração dos nutrientes, principalmente nas folhas; entretanto, estes valores de referência são geralmente estabelecidos em experimentos de calibração, em ambientes controlados (Bhargava \& Chadha, 1988), o que torna a identificação do estado nutricional restrita. Ocorre também que estes valores estão sujeitos a constantes revisões, em conseqüência da introdução de novos materiais, novas formas de cultivo e ambiente diferenciados, exigindo outros ensaios (Wadt et al., 1998a), o que, para culturas perenes, demandariam tempo e elevado gasto de recursos. Deste modo, uma alternativa prática e eficiente seria o uso de informações nutricionais associadas à respectiva produtividade de várias lavouras comerciais de determinada região e, com estes dados, obter valores de referência baseados em lavouras de alta produtividade (Beaufils, 1973); contudo, estes valores não podem ser utilizados para determinar curvas de resposta à adubação (Wadt et al., 1998a).

A análise de tecidos vegetais é vantajosa, pelo fato de ser a planta o extrator dos nutrientes no solo, possibilitando uma avaliação direta de seu estado nutricional (Beaufils, 1973). O Sistema Integrado de Diagnose e Recomendação (DRIS) preconizado por Beaufils (1973) incorpora o conceito de balanço nutricional ou de equilíbrio entre os nutrientes no tecido vegetal (Rathfon \& Burger, 1991). Esta técnica possibilita realizar o diagnóstico nutricional de uma planta e, ou, lavoura, com base no cálculo de índices para cada nutriente, avaliados de acordo com a relação das razões dos teores de cada elemento com os demais, comparando-os, dois a dois, com outras relações-padrão, obtidas de uma população de plantas altamente produtivas.

O índice DRIS possibilita definir o grau de desvio dos nutrientes da amostra e sua localização em relação ao estado nutricional, se adequado, em deficiência ou em excesso. Quanto mais próximo de zero estiver o índice do nutriente, mais próximo do equilíbrio nutricional o nutriente se encontra; índice positivo indica que o nutriente está em excesso e índice negativo, deficiente (Baldock \& Schulte, 1996).

As normas DRIS são úteis para estudar os desequilíbrios nutricionais e aumentar a produtividade da cultura, quando se realiza a correção do nutriente indicado no diagnóstico (Reis Júnior \& Monnerat, 2003b). Entretanto, fatores, tais como: déficit hídrico, genótipo cultivado, problemas fitossanitários, espaçamento e outros, podem influenciar o teor foliar e, principalmente, a produtividade da planta. Deste modo, o DRIS é um bom método para verificar desequilíbrio nutricional de uma planta e, ou, lavoura.

A afirmação de que a norma DRIS deve ser regional é relatado por Dara et al. (1992) e Reis Júnior \& Monnerat (2003a); entretanto, não há relatos indicando se estas normas proporcionam diagnósticos nutricionais semelhantes, em diferentes formas de cultivo, por exemplo, orgânico e convencional.

O cafeeiro conilon apresenta grande importância no agronegócio brasileiro e, atualmente, há um aumento no número de cultivos orgânicos; entretanto, 
faltam pesquisas específicas sobre esta forma de cultivo, principalmente sobre as questões nutricionais.

O objetivo deste trabalho foi estabelecer e comparar os padrões de referência e propor valores de teores foliares considerados adequados, obtidos a partir de lavouras de alta produtividade de cafeeiro conilon, cultivadas de forma orgânica ou convencional.

\section{MATERIAL E MÉTODOS}

As coletas de folhas foram efetuadas em lavouras de café conilon (Coffea canephora), cultivadas de forma orgânica ou convencional, localizadas no norte do Estado do Espírito Santo, onde predomina clima tropical, quente e úmido no verão e inverno seco com precipitação e temperatura média anual de $1.200 \mathrm{~mm}$ e de $23^{\circ} \mathrm{C}$ respectivamente. A região apresenta, em sua maioria, altitude entre 0 e $200 \mathrm{~m}$ (Espírito Santo, 1994).

Foram selecionadas 96 lavouras de café, sendo 40 sob o cultivo convencional (utilização de adubos altamente solúveis e agrotóxicos) e 56 sob o cultivo orgânico (certificadas ou em processo de certificação, nas quais não se utilizam agrotóxicos e, como fonte de nutrientes, são utilizados adubos orgânicos), com, no mínimo, 30 meses de conversão, no ato da colheita, tempo este superior ao necessário para a lavoura ser considerada orgânica (Brasil, 1999).

Foram realizados levantamentos de vários itens sobre as lavouras, dentre eles: insumos utilizados, idade, espaçamento entre plantas, número de hastes ortotrópicas por hectare, tipo de irrigação utilizada, existência de consórcio e outros. Posteriormente, coletaram-se aproximadamente 150 folhas em 50 plantas, por lavoura, distribuídas aleatoriamente. As folhas coletadas estavam situadas no terceiro e, ou, quarto nó do ramo plagiotrópico, a partir do ápice do ramo, localizado no terço mediano superior das plantas (Fullin \& Dadalto, 2001). A coleta foi realizada nos meses de dezembro de 2003 e janeiro de 2004. O teor de $\mathrm{N}$ foi obtido pelo método de Nessler, após a digestão do material seco com ácido sulfúrico e água oxigenada. $\mathrm{O}$ teor de $\mathrm{P}$ foi analisado colorimetricamente pelo método do molibdato. Quantificou-se o teor de K por fotometria de chama, os teores de $\mathrm{Ca}, \mathrm{Mg}, \mathrm{Mn}, \mathrm{Zn}, \mathrm{Fe}$ e $\mathrm{Cu}$, por espectrofotometria de absorção atômica, e o teor de $\mathrm{S}$, por turbidimetria, após digestão do material seco com ácido nítrico e ácido perclórico. O teor de $\mathrm{B}$ foi analisado por colorimetria com azometina- $\mathrm{H}$, depois da incineração do material vegetal em mufla a $550{ }^{\circ} \mathrm{C}$ (Malavolta et al., 1997).

Foi montado um banco de dados, separando as duas formas de cultivo e as lavouras de alta e baixa produtividade, com os teores foliares dos nutrientes e as respectivas produtividades. Foram consideradas lavouras de alta produtividade aquelas que apresentaram produtividade igual ou superior a $3.600 \mathrm{~kg} \mathrm{ha}^{-1}$ de café beneficiado, para as lavouras convencionais, e igual ou superior a $2.400 \mathrm{~kg} \mathrm{ha}^{-1}$, para as lavouras orgânicas. Produtividades de 2.400 ou $3.600 \mathrm{~kg} \mathrm{ha}^{-1}$ foram consideradas altas para o cafeeiro. Optou-se por este limite mais baixo $\left(2.400 \mathrm{~kg} \mathrm{ha}^{-1}\right)$ para o café orgânico, pois há poucos agricultores tecnificados na categoria e não haveria número suficiente de lavouras para o estabelecimento de normas, caso a produtividade fosse padronizada em $3.600 \mathrm{~kg} \mathrm{ha}^{-1}$.

Posteriormente, foi aplicado o teste de Lilliefors, estabelecendo o valor de $1 \%$, para verificar a normalidade dos valores referentes aos teores de cada nutriente, dos grupos de lavouras de alta produtividade, como o utilizado por Leite (1993). Este teste é usado para estudar variâncias estimadas e calculadas e não apresenta restrições a pequenas amostras (Dallal \& Wilkinson, 1986). As lavouras cujos teores de nutrientes seguiram distribuição normal foram usadas para estabelecer as normas DRIS (média, desvio-padrão e coeficiente de variação). Após este procedimento, foram calculadas, separadamente, as relações dos nutrientes das diferentes populações.

Para verificar as diferenças entre as normas estabelecidas, utilizou-se o teste "t" de Student que apresenta a capacidade de informar com segurança se há diferença ou não entre as médias de dois tratamentos (cultivo orgânico e convencional) e situações em que a variância é homogênea ou heterogênea. Os testes estatísticos foram realizados a $1 \%$.

Para comparar as duas normas estabelecidas (orgânica e convencional), foi realizado diagnóstico nutricional, obtendo-se os índices dos nutrientes, como é feito no M-DRIS, que, além de usar as relações dos teores dos nutrientes, como no DRIS, emprega também as concentrações dos nutrientes, comparando com as normas específicas (Hallmark et al., 1987). Quantificou-se o número de vezes que os nutrientes apresentaram índice mais negativo e mais positivo, nas lavouras orgânicas e convencionais, utilizando normas DRIS provenientes de lavouras orgânicas e de lavouras convencionais, estabelecidas neste trabalho.

Para o cálculo da relação normal reduzida dos teores de dois nutrientes, utilizou-se o método de Jones (1981), que é uma modificação da fórmula original de Beaufils (1973), mas apresentada na forma estudentizada. Utilizou-se este método por sua facilidade de operação (Alvarez V. \& Leite, 1999), pelo bom diagnóstico (Wadt et al., 1998a; Alvarez V. \& Leite, 1999), bem como por apresentar diagnósticos semelhantes aos de outros métodos (Wadt et al., 1998b), e, segundo Mourão Filho et al. (2002), ser superior aos métodos de Beaufils (1973) e Elwali \& Gascho (1984). 


\section{Método de Jones (1981):}

$$
f(A / B)=[(A / B)-(a / b)] k / s
$$

em que $f(A / B)$ é a função calculada da relação de nutrientes A e B; A/B é a relação de nutrientes da amostra a ser diagnosticada; $a / b$ e $s$ são a média e o desvio-padrão das relações $A / B$ de nutrientes da população utilizada para definir as normas; e $k$ é a constante de sensibilidade que, neste trabalho, foi igual a 10, usada para transformar os índices DRIS em números maiores.

O índice DRIS de um nutriente representa a média aritmética dos quocientes do teor de determinado nutriente com os teores dos demais nutrientes. Neste trabalho, foram utilizadas as relações diretas e inversas, como sugeridas por Alvarez V. \& Leite (1999), e todas as relações como as utilizadas por Bataglia \& Santos (1990) e Leite (1993).

$$
\begin{gathered}
\text { Índice } A=\{[f(A / B)+f(A / C)+\ldots \\
+f(A / N)]-[f(B / A)+f(C / A)+\ldots+f(N / A)]\} / n+m
\end{gathered}
$$

em que $n$ é o número de funções em que o nutriente $A$ aparece no numerador e $m$ o número de funções em que o nutriente $A$ aparece no denominador.

O índice de balanço nutricional - IBN (Sumner, 1977) consiste no somatório, em módulo, de todos os índices envolvidos e foi obtido pela seguinte expressão:

$$
I B N=[\text { índice } A]+[\text { índice } B]+\ldots+[\text { índice } N]
$$

\section{RESULTADOS E DISCUSSÃO}

Para estabelecer as normas DRIS, foram selecionadas 22 lavouras sob cultivo orgânico e 23 sob cultivo convencional, as quais apresentaram alta produtividade e cujos teores de nutrientes seguiram distribuição normal (Quadros 1 e 2), número este considerado suficiente, segundo Mourão Filho et al. (2002) e Reis Júnior \& Monnerat (2003b).

No quadro 1, estão descritos a média, o coeficiente de variação (CV) e o desvio-padrão de todas as relações entre os nutrientes, fornecendo suporte para fazer o diagnóstico por meio do DRIS de lavouras orgânicas ou convencionais, utilizando a fórmula de Beaufils (1973), ou Jones (1981), ou, ainda, a fórmula de Elwali \& Gascho (1984). Com a utilização dos dados descritos no quadro 2, pode-se, também realizar diagnóstico, por meio do M-DRIS (Hallmark et al., 1987) e fornecer uma referência dos teores considerados adequados dos respectivos nutrientes.

Por meio do Teste "t" a 1 \% (Quadro 1), podem-se verificar diferenças das relações de nutrientes, entre as duas formas de cultivo. Notam-se também (Quadro 2) diferenças entre teores médios dos nutrientes, confirmando que há diferenças entre as duas normas, sugerindo que estas, além de regionalizadas (Dara et al., 1992; Reis Júnior \& Monnerat, 2003a), devem ser específicas a determinada forma de cultivo, por exemplo orgânico ou convencional, visto que tais diferenças estão relacionadas com as diferentes práticas de manejo e, conseqüentemente, serão refletidas no estado nutricional do cafeeiro.

Não houve diferença entre o teor de $\mathrm{N}$ das duas normas (Quadro 2), uma vez que a disponibilidade de $\mathrm{N}$ para as plantas foi realizada em quantidades suficientes e equiparadas; as lavouras convencionais receberam, por ano, três ou quatro adubações com adubos químicos, simples ou com formulados, que continham $\mathrm{N}, \mathrm{P}$ e K, tendo a maioria das lavouras orgânicas recebido adubos orgânicos, estercos e compostos, os quais continham $\mathrm{N}$, além de outros nutrientes em sua constituição (Kiehl, 1985). Em muitas lavouras orgânicas, estavam sendo cultivadas nas entrelinhas do cafeeiro leguminosas que promoviam a fixação biológica de $\mathrm{N}$ atmosférico (Castro et al., 2004). Ocorreram, também, teores foliares semelhantes entre o $\mathrm{Mg}, \mathrm{Cu}, \mathrm{Fe}$ e $\mathrm{Mn}$, indicando que os teores foliares desses nutrientes não apresentaram alterações pelas diferentes formas de tratos culturais.

$\mathrm{O}$ teor foliar médio de $\mathrm{P}$ das lavouras orgânicas foi superior ao teor médio das lavouras convencionais (Quadro 2), possivelmente por ter sido a maioria das lavouras orgânicas adubada com formulação com P nos anos anteriores, e muitas, atualmente, receberem fosfato natural, matéria orgânica e rochas com altos teores de silicato. A adsorção do $\mathrm{P}$ pode ser menor com a aplicação de matéria orgânica, pois, segundo Rheinheimer et al. (2003), maior teor de matéria orgânica no solo pode diminuir a capacidade máxima de adsorção de $\mathrm{P}$ e a energia de ligação do fosfato aos grupos funcionais dos colóides inorgânicos do solo, fazendo com que o $\mathrm{P}$ fique mais disponível para as plantas. Fato semelhante ocorre com a utilização de silicato ou calcário mais silicatos, o qual, atua principalmente na diminuição da energia de ligação do fosfato (Smyth \& Senchez, 1980, citados por Novais \& Smyth, 1999).

O K apresenta maiores teores foliares nas lavouras sob cultivo convencional. Este fato pode estar relacionado com grandes aplicações de fórmulas que contêm cloreto de potássio; nas lavouras orgânicas, a principal fonte de K são os restos provenientes do beneficiamento do café, e este material nem sempre é disponibilizado em quantidades equivalentes às aplicações de $\mathrm{K}$ das lavouras convencionais.

As lavouras cultivadas de forma orgânica apresentaram teor foliar médio de $\mathrm{S}$ superior ao das lavouras convencionais. Esta diferença pode estar relacionada com a adição de resíduos orgânicos, que, posteriormente, são mineralizada, ocorrendo a 
Quadro 1. Média, desvio-padrão e coeficiente de variação (CV) das relações dos teores de nutrientes das lavouras de café conilon de alta produtividade, sob o cultivo orgânico ou convencional, e a verificação da diferença entre elas pelo teste " $t$ " de Student

\begin{tabular}{|c|c|c|c|c|c|c|c|}
\hline \multirow{2}{*}{ Relação } & \multicolumn{3}{|c|}{ Lavouras orgânicas } & \multicolumn{3}{|c|}{ Lavouras convencionais ${ }^{(1)}$} & \multirow{2}{*}{ Teste $t$} \\
\hline & Média & Desvio-padrão & CV & Média & Desvio-padrão & $\mathrm{CV}$ & \\
\hline $\mathrm{N} / \mathrm{P}$ & 16,99 & 1,492 & 8,78 & 18,79 & 2,482 & 13,21 & $* *$ \\
\hline $\mathrm{N} / \mathrm{K}$ & 1,676 & 0,188 & 11,19 & 1,444 & 0,152 & 10,53 & $* *$ \\
\hline $\mathrm{N} / \mathrm{Ca}$ & 2,059 & 0,181 & 8,80 & 2,338 & 0,380 & 16,25 & $* *$ \\
\hline $\mathrm{N} / \mathrm{Mg}$ & 8,037 & 1,496 & 18,61 & 7,538 & 0,985 & 13,07 & NS \\
\hline $\mathrm{N} / \mathrm{S}$ & 13,52 & 1,817 & 13,44 & 15,45 & 3,007 & 19,47 & NS \\
\hline N/B & 0,522 & 0,108 & 20,6 & 0,429 & 0,058 & 13,54 & $* *$ \\
\hline $\mathrm{N} / \mathrm{Cu}$ & 1,893 & 0,647 & 34,17 & 2,509 & 0,995 & 39,63 & NS \\
\hline $\mathrm{N} / \mathrm{Fe}$ & 0,283 & 0,113 & 39,83 & 0,251 & 0,027 & 10,73 & NS \\
\hline $\mathrm{N} / \mathrm{Mn}$ & 0,431 & 0,208 & 48,19 & 0,382 & 0,174 & 45,42 & NS \\
\hline $\mathrm{N} / \mathrm{Zn}$ & 3,132 & 0,304 & 9,71 & 2,465 & 0,314 & 12,74 & $* * *$ \\
\hline $\mathrm{P} / \mathrm{N}$ & 0,059 & 0,005 & 8,58 & 0,054 & 0,007 & 12,86 & $* *$ \\
\hline $\mathrm{P} / \mathrm{K}$ & 0,099 & 0,014 & 14,37 & 0,078 & 0,010 & 12,30 & $* *$ \\
\hline $\mathrm{P} / \mathrm{Ca}$ & 0,122 & 0,014 & 11,58 & 0,126 & 0,022 & 17,44 & NS \\
\hline $\mathrm{P} / \mathrm{Mg}$ & 0,475 & 0,092 & 19,35 & 0,408 & 0,076 & 18,65 & NS \\
\hline $\mathrm{P} / \mathrm{S}$ & 0,798 & 0,103 & 12,94 & 0,829 & 0,153 & 18,41 & NS \\
\hline $\mathrm{P} / \mathrm{B}$ & 0,031 & 0,006 & 20,98 & 0,023 & 0,005 & 19,85 & $* *$ \\
\hline $\mathrm{P} / \mathrm{Cu}$ & 0,110 & 0,031 & 28,19 & 0,135 & 0,051 & 38,15 & NS \\
\hline $\mathrm{P} / \mathrm{Fe}$ & 0,017 & 0,007 & 40,15 & 0,014 & 0,002 & 16,79 & NS \\
\hline $\mathrm{P} / \mathrm{Mn}$ & 0,026 & 0,013 & 49,83 & 0,021 & 0,010 & 47,33 & NS \\
\hline $\mathrm{P} / \mathrm{Zn}$ & 0,186 & 0,026 & 13,91 & 0,132 & 0,017 & 12,54 & $* *$ \\
\hline $\mathrm{K} / \mathrm{N}$ & 0,604 & 0,069 & 11,42 & 0,700 & 0,076 & 10,89 & $* *$ \\
\hline $\mathrm{K} / \mathrm{P}$ & 10,25 & 1,404 & 13,69 & 13,07 & 1,554 & 11,89 & $* *$ \\
\hline $\mathrm{K} / \mathrm{Ca}$ & 1,245 & 0,186 & 14,95 & 1,625 & 0,245 & 15,08 & $* *$ \\
\hline $\mathrm{K} / \mathrm{Mg}$ & 4,867 & 1,088 & 22,35 & 5,292 & 0,958 & 18,10 & NS \\
\hline $\mathrm{K} / \mathrm{S}$ & 8,138 & 1,272 & 15,63 & 10,73 & 1,847 & 17,22 & $* *$ \\
\hline K/B & 0,315 & 0,07 & 22,36 & 0,300 & 0,048 & 15,85 & NS \\
\hline $\mathrm{K} / \mathrm{Cu}$ & 1,125 & 0,332 & 29,54 & 1,734 & 0,610 & 35,16 & $* *$ \\
\hline $\mathrm{K} / \mathrm{Fe}$ & 0,175 & 0,086 & 48,99 & 0,176 & 0,026 & 14,60 & NS \\
\hline $\mathrm{K} / \mathrm{Mn}$ & 0,263 & 0,142 & 53,91 & 0,265 & 0,114 & 43,07 & NS \\
\hline $\mathrm{K} / \mathrm{Zn}$ & 1,902 & 0,351 & 18,47 & 1,716 & 0,212 & 12,38 & NS \\
\hline $\mathrm{Ca} / \mathrm{N}$ & 0,489 & 0,043 & 8,85 & 0,440 & 0,079 & 18,02 & NS \\
\hline $\mathrm{Ca} / \mathrm{P}$ & 8,299 & 0,898 & 10,82 & 8,205 & 1,524 & 18,57 & NS \\
\hline $\mathrm{Ca} / \mathrm{K}$ & 0,822 & 0,131 & 15,97 & 0,630 & 0,107 & 19,99 & $* *$ \\
\hline $\mathrm{Ca} / \mathrm{Mg}$ & 3,898 & 0,614 & 15,75 & 3,308 & 0,710 & 21,46 & NS \\
\hline $\mathrm{Ca} / \mathrm{S}$ & 6,585 & 0,853 & 12,95 & 6,722 & 1,446 & 21,51 & NS \\
\hline $\mathrm{Ca} / \mathrm{B}$ & 0,252 & 0,037 & 14,48 & 0,188 & 0,039 & 20,72 & $* *$ \\
\hline $\mathrm{Ca} / \mathrm{Cu}$ & 0,916 & 0,305 & 33,23 & 1,075 & 0,379 & 35,26 & NS \\
\hline $\mathrm{Ca} / \mathrm{Fe}$ & 0,137 & 0,052 & 38,22 & 0,111 & 0,025 & 22,46 & NS \\
\hline $\mathrm{Ca} / \mathrm{Mn}$ & 0,208 & 0,095 & 45,55 & 0,163 & 0,069 & 42,19 & NS \\
\hline $\mathrm{Ca} / \mathrm{Zn}$ & 1,536 & 0,235 & 15,32 & 1,083 & 0,244 & 22,55 & $* *$ \\
\hline $\mathrm{Mg} / \mathrm{N}$ & 0,129 & 0,024 & 19,05 & 0,135 & 0,019 & 14,00 & NS \\
\hline $\mathrm{Mg} / \mathrm{P}$ & 2,175 & 0,38 & 17,45 & 2,543 & 0,540 & 21,23 & NS \\
\hline $\mathrm{Mg} / \mathrm{K}$ & 0,217 & 0,055 & 25,23 & 0,196 & 0,039 & 19,79 & NS \\
\hline $\mathrm{Mg} / \mathrm{Ca}$ & 0,262 & 0,040 & 15,36 & 0,315 & 0,063 & 19,89 & $* *$ \\
\hline $\mathrm{Mg} / \mathrm{S}$ & 1,724 & 0,324 & 18,76 & 2,073 & 0,436 & 21,02 & $* *$ \\
\hline $\mathrm{Mg} / \mathrm{B}$ & 0,066 & 0,01 & 14,99 & 0,058 & 0,010 & 18,07 & $* *$ \\
\hline $\mathrm{Mg} / \mathrm{Cu}$ & 0,237 & 0,078 & 32,7 & 0,337 & 0,138 & 40,90 & NS \\
\hline $\mathrm{Mg} / \mathrm{Fe}$ & 0,037 & 0,016 & 44,22 & 0,034 & 0,005 & 16,21 & NS \\
\hline $\mathrm{Mg} / \mathrm{Mn}$ & 0,055 & 0,029 & 52,3 & 0,051 & 0,022 & 42,61 & NS \\
\hline $\mathrm{Mg} / \mathrm{Zn}$ & 0,404 & 0,094 & 23,35 & 0,334 & 0,075 & 22,48 & $* *$ \\
\hline $\mathrm{S} / \mathrm{N}$ & 0,075 & 0,011 & 14,5 & 0,067 & 0,014 & 20,43 & NS \\
\hline $\mathrm{S} / \mathrm{P}$ & 1,274 & 0,169 & 13,27 & 1,253 & 0,284 & 22,62 & NS \\
\hline $\mathrm{S} / \mathrm{K}$ & 0,126 & 0,020 & 15,94 & 0,096 & 0,020 & 20,56 & $* *$ \\
\hline $\mathrm{S} / \mathrm{Ca}$ & 0,154 & 0,020 & 13,37 & 0,155 & 0,034 & 21,79 & NS \\
\hline $\mathrm{S} / \mathrm{Mg}$ & 0,600 & 0,116 & 19,28 & 0,503 & 0,104 & 20,74 & NS \\
\hline $\mathrm{S} / \mathrm{B}$ & 0,039 & 0,006 & 16,07 & 0,029 & 0,007 & 23,60 & $* * *$ \\
\hline $\mathrm{S} / \mathrm{Cu}$ & 0,139 & 0,04 & 28,52 & 0,166 & 0,065 & 39,26 & NS \\
\hline $\mathrm{S} / \mathrm{Fe}$ & 0,021 & 0,01 & 44,76 & 0,017 & 0,004 & 21,90 & NS \\
\hline $\mathrm{S} / \mathrm{Mn}$ & 0,033 & 0,02 & 61,59 & 0,026 & 0,013 & 49,85 & NS \\
\hline $\mathrm{S} / \mathrm{Zn}$ & 0,237 & 0,05 & 21,01 & 0,165 & 0,039 & 23,68 & $* *$ \\
\hline
\end{tabular}

Continua... 
Quadro 1. Continuação

\begin{tabular}{|c|c|c|c|c|c|c|c|}
\hline \multirow{2}{*}{ Relação } & \multicolumn{3}{|c|}{ Lavouras orgânicas } & \multicolumn{3}{|c|}{ Lavouras convencionais ${ }^{(1)}$} & \multirow{2}{*}{ Teste $t$} \\
\hline & Média & Desvio-padrão & CV & Média & Desvio-padrão & CV & \\
\hline $\mathrm{B} / \mathrm{N}$ & 1,989 & 0,387 & 19,47 & 2,371 & 0,322 & 13,59 & $* *$ \\
\hline $\mathrm{B} / \mathrm{P}$ & 33,61 & 6,028 & 17,94 & 44,45 & 7,452 & 16,77 & $* *$ \\
\hline $\mathrm{B} / \mathrm{K}$ & 3,336 & 0,789 & 23,67 & 3,413 & 0,515 & 15,08 & NS \\
\hline $\mathrm{B} / \mathrm{Ca}$ & 4,047 & 0,616 & 15,22 & 5,517 & 1,022 & 18,52 & $* *$ \\
\hline $\mathrm{B} / \mathrm{Mg}$ & 15,61 & 2,502 & 16,03 & 17,82 & 3,066 & 17,20 & $* *$ \\
\hline $\mathrm{B} / \mathrm{S}$ & 26,45 & 3,934 & 14,89 & 36,47 & 7,829 & 21,47 & $* *$ \\
\hline $\mathrm{B} / \mathrm{Cu}$ & 3,669 & 1,234 & 33,65 & 5,813 & 2,063 & 35,49 & $* *$ \\
\hline $\mathrm{B} / \mathrm{Fe}$ & 0,553 & 0,222 & 40,18 & 0,596 & 0,106 & 17,74 & NS \\
\hline $\mathrm{B} / \mathrm{Mn}$ & 0,832 & 0,378 & 45,45 & 0,902 & 0,408 & 45,21 & NS \\
\hline $\mathrm{B} / \mathrm{Zn}$ & 6,262 & 1,468 & 23,45 & 5,848 & 1,085 & 18,55 & NS \\
\hline $\mathrm{Cu} / \mathrm{N}$ & 0,589 & 0,194 & 32,95 & 0,456 & 0,165 & 36,22 & NS \\
\hline $\mathrm{Cu} / \mathrm{P}$ & 9,818 & 2,802 & 28,54 & 8,552 & 3,473 & 40,61 & NS \\
\hline $\mathrm{Cu} / \mathrm{K}$ & 0,977 & 0,334 & 34,24 & 0,654 & 0,249 & 38,12 & $* *$ \\
\hline $\mathrm{Cu} / \mathrm{Ca}$ & 1,195 & 0,343 & 28,71 & 1,042 & 0,361 & 34,66 & NS \\
\hline $\mathrm{Cu} / \mathrm{Mg}$ & 4,599 & 1,3 & 28,27 & 3,402 & 1,173 & 34,47 & $* *$ \\
\hline $\mathrm{Cu} / \mathrm{S}$ & 7,799 & 2,369 & 30,38 & 6,931 & 2,583 & 37,27 & NS \\
\hline $\mathrm{Cu} / \mathrm{B}$ & 0,298 & 0,084 & 28,32 & 0,191 & 0,061 & 31,78 & $* *$ \\
\hline $\mathrm{CuFe}$ & 0,173 & 0,101 & 58,56 & 0,116 & 0,046 & 39,95 & NS \\
\hline $\mathrm{Cu} / \mathrm{Mn}$ & 0,247 & 0,131 & 52,97 & 0,162 & 0,068 & 41,76 & NS \\
\hline $\mathrm{Cu} / \mathrm{Zn}$ & 1,870 & 0,723 & 38,65 & 1,135 & 0,505 & 44,50 & $* *$ \\
\hline $\mathrm{Fe} / \mathrm{N}$ & 4,100 & 1,613 & 39,33 & 4,025 & 0,431 & 10,71 & NS \\
\hline $\mathrm{Fe} / \mathrm{P}$ & 69,75 & 28,68 & 41,12 & 75,58 & 12,55 & 16,61 & NS \\
\hline $\mathrm{Fe} / \mathrm{K}$ & 6,947 & 2,826 & 40,68 & 5,813 & 0,913 & 15,70 & NS \\
\hline $\mathrm{Fe} / \mathrm{Ca}$ & 8,357 & 3,175 & 37,98 & 9,411 & 1,784 & 18,96 & NS \\
\hline $\mathrm{Fe} / \mathrm{Mg}$ & 33,08 & 14,9 & 45,05 & 30,31 & 5,056 & 16,68 & NS \\
\hline $\mathrm{Fe} / \mathrm{S}$ & 55,23 & 21,89 & 39,64 & 62,02 & 12,81 & 20,66 & NS \\
\hline $\mathrm{Fe} / \mathrm{B}$ & 2,095 & 0,793 & 37,85 & 1,730 & 0,311 & 17,98 & NS \\
\hline $\mathrm{Fe} / \mathrm{Cu}$ & 7,981 & 4,399 & 55,12 & 10,24 & 4,740 & 46,30 & NS \\
\hline $\mathrm{Fe} / \mathrm{Mn}$ & 1,652 & 0,679 & 41,07 & 1,535 & 0,684 & 44,56 & NS \\
\hline $\mathrm{Fe} / \mathrm{Zn}$ & 12,75 & 5,099 & 39,99 & 9,922 & 1,625 & 16,37 & NS \\
\hline $\mathrm{Mn} / \mathrm{N}$ & 2,691 & 0,929 & 34,51 & 3,214 & 1,545 & 48,07 & NS \\
\hline $\mathrm{Mn} / \mathrm{P}$ & 45,72 & 15,72 & 34,38 & 60,89 & 32,60 & 53,53 & NS \\
\hline $\mathrm{Mn} / \mathrm{K}$ & 4,521 & 1,641 & 36,3 & 4,617 & 2,246 & 48,65 & NS \\
\hline $\mathrm{Mn} / \mathrm{Ca}$ & 5,476 & 1,767 & 32,26 & 7,332 & 3,469 & 47,31 & NS \\
\hline $\mathrm{Mn} / \mathrm{Mg}$ & 21,60 & 8,471 & 39,22 & 24,00 & 12,08 & 50,33 & NS \\
\hline $\mathrm{Mn} / \mathrm{S}$ & 36,24 & 12,18 & 33,6 & 49,45 & 25,27 & 51,10 & NS \\
\hline $\mathrm{Mn} / \mathrm{B}$ & 1,368 & 0,464 & 33,87 & 1,382 & 0,717 & 51,89 & NS \\
\hline $\mathrm{Mn} / \mathrm{Cu}$ & 4,952 & 2,037 & 41,14 & 7,508 & 3,628 & 48,32 & $* *$ \\
\hline $\mathrm{Mn} / \mathrm{Fe}$ & 0,730 & 0,341 & 46,7 & 0,813 & 0,434 & 53,43 & NS \\
\hline $\mathrm{Mn} / \mathrm{Zn}$ & 8,394 & 2,897 & 34,52 & 7,935 & 4,024 & 50,71 & NS \\
\hline $\mathrm{Zn} / \mathrm{N}$ & 0,322 & 0,032 & 9,83 & 0,412 & 0,050 & 12,29 & $* *$ \\
\hline $\mathrm{Zn} / \mathrm{P}$ & 5,486 & 0,829 & 15,12 & 7,676 & 0,969 & 12,63 & $* *$ \\
\hline $\mathrm{Zn} / \mathrm{K}$ & 0,543 & 0,100 & 18,38 & 0,592 & 0,076 & 12,81 & $\mathrm{NS}$ \\
\hline $\mathrm{Zn} / \mathrm{Ca}$ & 0,665 & 0,099 & 14,9 & 0,960 & 0,177 & 18,42 & $* *$ \\
\hline $\mathrm{Zn} / \mathrm{Mg}$ & 2,598 & 0,576 & 22,16 & 3,114 & 0,587 & 18,85 & $* *$ \\
\hline $\mathrm{Zn} / \mathrm{S}$ & 4,381 & 0,862 & 19,68 & 6,339 & 1,346 & 21,24 & $* *$ \\
\hline $\mathrm{Zn} / \mathrm{B}$ & 0,170 & 0,045 & 26,78 & 0,177 & 0,035 & 19,82 & $\mathrm{NS}$ \\
\hline $\mathrm{Zn} / \mathrm{Cu}$ & 0,619 & 0,249 & 40,19 & 1,034 & 0,416 & 40,26 & $* *$ \\
\hline $\mathrm{Zn} / \mathrm{Fe}$ & 0,090 & 0,032 & 36,61 & 0,104 & 0,018 & 17,14 & NS \\
\hline $\mathrm{Zn} / \mathrm{Mn}$ & 0,138 & 0,064 & 46,54 & 0,157 & 0,070 & 44,42 & NS \\
\hline
\end{tabular}

NS e **: Não-significativo e significativo a $1 \%$.

restituição do S (Kiehl, 1985); estercos de suínos e bovinos contêm $\mathrm{S}$ em sua composição. Outro fato que pode ter contribuído com teores mais baixos de $\mathrm{S}$ nas lavouras convencionais é que estas, geralmente, não recebem adubos simples, como sulfato de amônio, superfosfato simples e, ou, formulados com S.

Verificou-se que o teor foliar de Ca em lavouras convencionais foi mais baixo, em relação às lavouras orgânicas (Quadro 2). Este fato pode estar relacionado com o antagonismo de $\mathrm{K}$ com o $\mathrm{Ca}$, em que o excesso do primeiro ocasiona a deficiência do segundo (Marschner, 1995), visto que as lavouras convencionais receberam elevadas aplicações de $\mathrm{K}$.

Os nutrientes B e Zn apresentaram maior teor foliar médio nas lavouras convencionais (Quadro 2). Provavelmente, isto deveu-se à adição de adubos 
formulados que continham B e Zn. No caso do Zn, pode ocorrer também antagonismo com o $\mathrm{P}$, já que, segundo Marschner (1995), um aumento no suprimento de $\mathrm{P}$ poderia levar à redução na absorção e, ou, diluição foliar do Zn, quando este apresenta baixo teor no solo.

Comparando os diagnósticos nutricionais nas lavouras orgânicas e convencionais, utilizando normas orgânicas e normas convencionais estabelecidas para ambos os grupos de lavouras, pelas freqüências do nutriente, com o índice DRIS sendo mais negativo ou mais positivo (Quadro 3), houve grande diferença entre os diagnósticos, ao utilizar normas distintas. Por exemplo, a ordem de freqüência do nutriente com índice mais negativo nas lavouras orgânicas foi $\mathrm{Mn}, \mathrm{Cu}, \mathrm{N}, \mathrm{P}, \mathrm{Fe}, \mathrm{K}=\mathrm{Zn}=\mathrm{Mg}, \mathrm{B}$ e Ca e nenhuma para o S, utilizando normas orgânicas. Entretanto, ao utilizar normas convencionais, verificou-se que o $\mathrm{Fe}, \mathrm{Zn}, \mathrm{K}, \mathrm{B}, \mathrm{Mn}, \mathrm{Mg}$ e $\mathrm{Cu}$, apareceram com maior freqüência, enquanto o N, P, Ca e S não apareceram nenhuma vez como o nutriente com índice mais negativo. Nas lavouras convencionais, a maior freqüência de nutrientes com índice mais negativo foi $\mathrm{Mn}$, $\mathrm{Cu}$ e P, ao utilizar normas convencionais; no entanto, ao utilizar normas orgânicas, foi $\mathrm{Cu}, \mathrm{Ca}, \mathrm{P}$ e S.

Quadro 2. Média, desvio-padrão e coeficiente de variação (CV) dos teores de nutrientes das lavouras de café conilon de alta produtividade, sob o cultivo orgânico ou convencional, e a verificação da diferença entre os teores de nutrientes pelo teste "t" de Student

\begin{tabular}{|c|c|c|c|c|c|c|c|}
\hline \multirow{2}{*}{ Nutriente } & \multicolumn{3}{|c|}{ Lavouras orgânicas } & \multicolumn{3}{|c|}{ Lavouras convencionais ${ }^{(1)}$} & \multirow{2}{*}{ Teste } \\
\hline & Média & Desvio-padrão & CV & Média & Desvio-padrão & CV & \\
\hline $\mathrm{N}\left(\mathrm{g} \mathrm{kg}^{-1}\right)$ & 27,6 & 1,43 & 5,2 & 26,4 & 1,5 & 5,8 & NS \\
\hline $\mathrm{P}\left(\mathrm{g} \mathrm{kg}^{-1}\right)$ & 1,6 & 0,12 & 7,4 & 1,4 & 0,17 & 12,1 & $* *$ \\
\hline $\mathrm{K}\left(\mathrm{g} \mathrm{kg}^{-1}\right)$ & 16,7 & 2,0 & 12 & 18,5 & 1,7 & 9,1 & $* *$ \\
\hline $\mathrm{Ca}\left(\mathrm{g} \mathrm{kg}^{-1}\right)$ & 13,5 & 1,13 & 8,4 & 11,6 & 2,2 & 19,2 & $* *$ \\
\hline $\mathrm{Mg}\left(\mathrm{g} \mathrm{kg}^{-1}\right)$ & 3,5 & 0,62 & 17,6 & 3,6 & 0,48 & 13,5 & NS \\
\hline $\mathrm{S}\left(\mathrm{g} \mathrm{kg}^{-1}\right)$ & 2,1 & 0,24 & 11,6 & 1,8 & 0,34 & 19,0 & $* *$ \\
\hline $\mathrm{B}\left(\mathrm{mg} \mathrm{kg}^{-1}\right)$ & 54,5 & 8,9 & 16,5 & 62,4 & 7,2 & 11,6 & $* *$ \\
\hline $\mathrm{Cu}\left(\mathrm{mg} \mathrm{kg}^{-1}\right)$ & 16,1 & 5,0 & 31,2 & 12 & 4,3 & 35,7 & $\mathrm{NS}$ \\
\hline $\mathrm{Fe}\left(\mathrm{mg} \mathrm{kg}^{-1}\right)$ & 112 & 42,8 & 38,1 & 106 & 11,9 & 11,2 & NS \\
\hline $\operatorname{Mn}\left(\mathrm{mg} \mathrm{kg}^{-1}\right)$ & 73,9 & 24,3 & 32,9 & 85 & 41,9 & 49,3 & $\mathrm{NS}$ \\
\hline $\mathrm{Zn}\left(\mathrm{mg} \mathrm{kg}^{-1}\right)$ & 8,9 & 1,1 & 12,1 & 10,9 & 1,5 & 13,3 & $* *$ \\
\hline
\end{tabular}

NS e **: Não-significativo e significativo a $1 \%$.

Quadro 3. Freqüência de nutriente com índice DRIS mais negativo (+ NE) e mais positivo (+ PO), nas 56 lavouras orgânicas e nas 40 lavouras convencionais, utilizando normas orgânicas (Normas Org.) e normas convencionais (Normas Conv.) da região, para ambos os grupos de lavouras, por meio das normas DRIS com incorporação da matéria seca (M-DRIS)

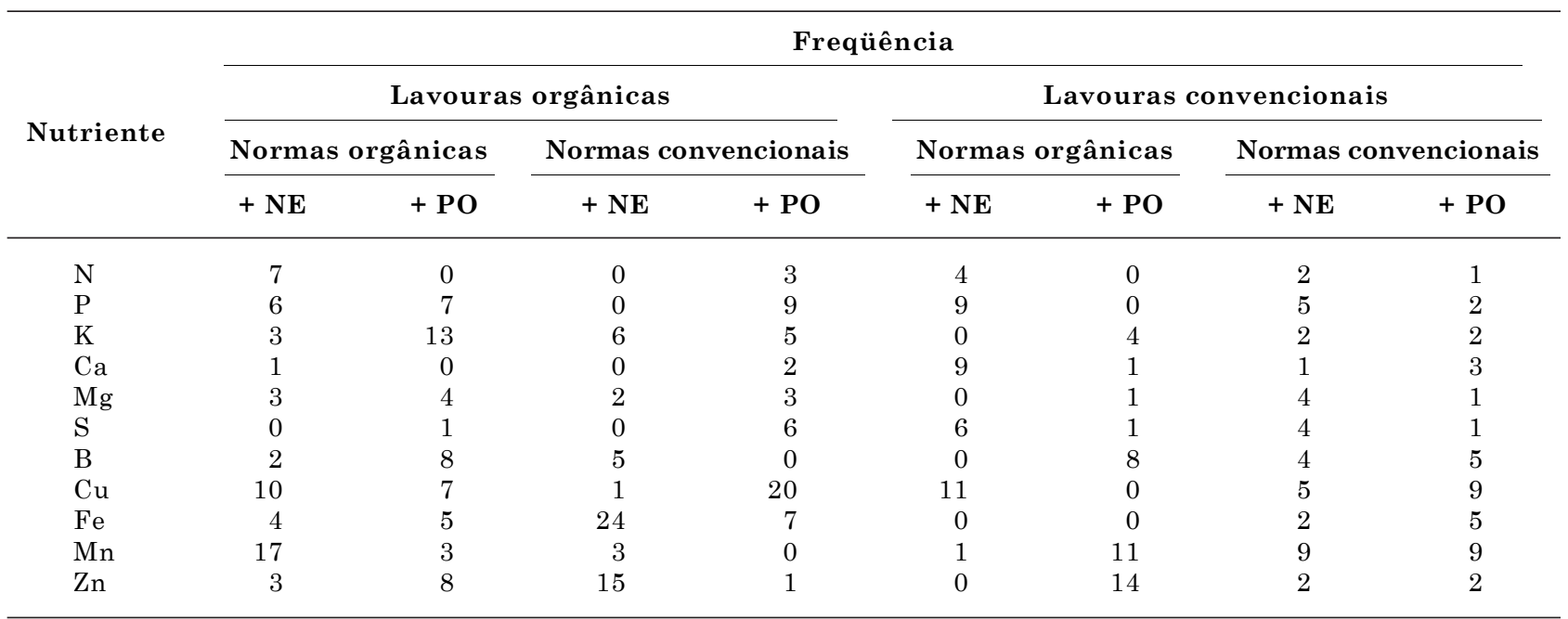


Assim, o diagnóstico de lavouras orgânicas utilizando normas convencionais e de lavouras convencionais utilizando normas orgânicas pode ser indesejável, por fornecer diagnóstico bem diferente daquele que utiliza as normas específicas. Assim, esta diferença entre diagnósticos indica que as normas devem ser específicas a determinada forma de cultivo.

\section{CONCLUSÕES}

1. Os nutrientes que apresentaram índices DRIS mais negativos nas lavouras orgânicas foram $\mathrm{Mn}, \mathrm{Cu}$, $\mathrm{N}$ e $\mathrm{P}$, e nas lavouras convencionais foram $\mathrm{Mn}, \mathrm{Cu}$ e $\mathrm{P}$.

2. Houve diferença em 41 relações de nutrientes dentre as 110, das duas formas de cultivo.

3. Os teores médios dos nutrientes $\mathrm{P}, \mathrm{Ca}$ e $\mathrm{S}$ foram maiores no cultivo orgânico, enquanto o K, B e Zn foram maiores no cultivo convencional.

4. Sugere-se que as normas DRIS sejam específicas a cada forma de cultivo, orgânico ou convencional; caso contrário, os diagnósticos podem ser incorretos.

\section{AGRADECIMENTOS}

Os autores agradecem à Universidade Estadual do Norte Fluminense Darcy Ribeiro - UENF, Fundação de Amparo à Pesquisa do Estado do Rio de Janeiro - FAPERJ, MIBASA - Mineração Barreto, Instituto Capixaba de Pesquisa Assistência Técnica e Extensão Rural - INCAPER, BIOSYSTEMS e aos agricultores.

\section{LITERATURA CITADA}

ALVAREZ V., V.H. \& LEITE, R.A. Fundamentos estatísticos das fórmulas usadas para cálculo dos índices DRIS: R. B. Inf. SBSC, 24:20-25, 1999.

BALDOCK, J.O. \& SCHULTE, E.E. Plant analysis with standardized scores combines DRIS and sufficiency range approaches for corn. Agron. J., 88:448-456, 1996.

BATAGLIA, O.C. \& SANTOS, W.R. Efeito do procedimento de cálculo e da população de referência nos índices do sistema integrado de diagnose e recomendação (DRIS). R. Bras. Ci. Solo, 14:339-344, 1990.

BEAUFILS, E.R. Diagnosis and recommendation integrated system (DRIS). A general scheme of experimentation and calibration based on principles developed from research in plant nutrition. South Africa, University of Natal, Pietermaritzburg, 1973. 132p. (Soil Science Bulletin, 1)
BHARGAVA, B.S. \& CHADHA, K.L. Leaf nutrient guide for fruit and plantation crops. Fert. News, 33:21-29, 1988.

BRASIL. Ministério da Agricultura e do Abastecimento. Instrução Normativa n. 7, de 17 de maio de 1999. Dispõe sobre normas para a produção de produtos orgânicos vegetais e animais. Diário Oficial [da] República Federativa do Brasil, Poder Executivo, Brasília, DF, 19 de maio 1999. Seção 1.

CASTRO, C.M.; ALVES, B.J.R.; ALMEIDA, D.L. \& RIBEIRO, R.L.D. Adubação verde como fonte de nitrogênio para a cultura da berinjela em sistema orgânico. Pesq. Agropec. Bras., 39:779-785, 2004.

DARA, S.T.; FIXEN, P.E. \& GELDERMAN, R.H. Sufficiency level and diagnosis and recommendation integrated system approaches for evaluating the nitrogen status of the corn. Agron. J., 84:1006-1010, 1992.

ELWALI, A.M.O. \& GASCHO, G.J. Soil testing, foliar analysis and DRIS as guides for sugarcane fertilization. Agron. J., 76:466-470, 1984.

ESPÍRITO SANTO (Estado). Secretaria de Estado de Ações Estratégicas e Planejamento. Informações Municipais do Estado do Espírito Santo 1994. Vitória, Departamento Estadual de Estatística, 1994. v. 1. 803p.

DALLAL, G. E. \& WILKINSON, L. An analytic approximation to the distribution of 'Lilliefors' test for normality. The Amer. Statist., 40:294-296, 1986.

FULLIN, E.A. \& DADALTO, G.G. Avaliação da fertilidade do solo e do estado nutricional das plantas. In: DADALTO, G.G. \& FULLIN, E.A., eds. Manual de recomendação de calagem e adubação para o Estado do Espírito Santo: quarta aproximação. Vitória, SEEA/INCAPER, 2001. p. 21-55.

HALLMARK, W.B.; MOOY, C.J. \& PESEK, J. Comparison of two DRIS methods for diagnosing nutrient deficiencies. J. Fert. Issues, 4:151-158, 1987.

JONES, W.W. Proposed modifications of the diagnosis and recommendation integrated system (DRIS) for interpreting plant analyses. Comm. Soil Sci. Plant Anal., 12:785-794, 1981.

KIEHL, E.J. Fertilizantes orgânicos. São Paulo, Agronômica Ceres, 1985. 492p.

LEITE, R.A. Avaliação do estado nutricional do cafeeiro conilon no Estado do Espírito Santo utilizando diferentes métodos de interpretação de análise foliar. Viçosa, MG, Universidade Federal de Viçosa, 1993. 87p. (Tese de Doutorado)

MARSCHNER, H. Mineral nutrition of higher plants. London, Academic Press, 1995. 889p.

MALAVOLTA, E.; VITTI, G.C. \& OLIVEIRA, S.A. Avaliação do estado nutricional das plantas: princípios e aplicações. Piracicaba, Potafos, 1997. 319p.

MOURÃO FILHO, F.A.; AZEVEDO, J.C. \& NICK, J.A. Funções e ordem da razão dos nutrientes no estabelecimento de normas DRIS em laranjeira "Valência". Pesq. Agropec. Bras., 37:185-192, 2002. 
NOVAIS, R.F. \& SMYTH, T.J. Fósforo em solo e planta em condições tropicais. Viçosa, MG, Universidade Federal de Viçosa, 1999. 399p.

RATHFON, R.A. \& BURGER, J.A. Diagnosis and recommendation integrated system modifications for Fraser Fir Christmas trees. Amer. J., 55:1026-1031, 1991.

REIS JÚNIOR, R.A. \& MONNERAT, P.H. Norms establishment of the diagnosis and recommendation integrated system (DRIS) for nutritional diagnosis of sugarcane. Pes. Agropec. Bras., 38:277-282, $2003 a$.

REIS JÚNIOR, R.A. \& MONNERAT, P.H. DRIS norms validation for sugarcane crop. Pesq. Agropec. Bras., 38:379$385,2003 b$.
RHEINHEINER, D.S.; ANGHINONI, I. \& CONTE, E. Sorção de fósforo em função do teor inicial e de sistemas de manejo de solos. R. Bras. Ci. Solo, 27:41-49, 2003.

SUMNER, M.E. Use of the DRIS system in foliar diagnosis of crops at high yield levels. Comm. Soil Sci. Plant Anal., 8:251-268, 1977.

WADT, P.G.S.; NOVAIS, R.F.; ALVAREZ V., V.H.; FONSECA, S. \& BARROS, N.F. Valores de referência para macronutrientes em eucalipto obtidos pelos métodos DRIS e chance matemática. R. Bras. Ci. Solo, 22:685-692, 1998a.

WADT, P.G.S.; NOVAIS, R.F.; ALVAREZ V., V.H.; FONSECA, S. \& BARROS, N.F. Três métodos de cálculo do DRIS para avaliar o potencial de resposta à adubação de árvores de eucalipto. R. Bras. Ci. Solo, 22:651-660, 1998b. 